\title{
Antibiotic Therapy of Fulminant E. Coli K1 Sepsis in Infant Rabbits
}

\author{
BARBARA J. LAW, ${ }^{(27)}$ PHILIP J. RETTIG, AND MELVIN I. MARKS ${ }^{(28)}$ \\ Department of Pediatrics (Infectious Diseases), University of Oklahoma, Health Sciences Center, \\ Oklahoma City, Oklahoma, USA
}

\begin{abstract}
Summary
A model of overwhelming $E$. coli $\mathrm{K} 1$ sepsis and early meningitis was developed in infant rabbits and used to compare clinical and bacteriologic efficacy of ampicillin, moxalactam, cephalothin and chloramphenicol. Intraperitoneal injection of $10^{7} E$. coli $\mathrm{K} 1$ into 1- or 2-wk-old rabbits produced a rapidly progressive infection which, if left untreated, produced bacteremia in $100 \%$ of animals, meningitis in $78 \%$, and mortality in $100 \%$. Therapy was initiated $4 \mathrm{~h}$ after ip infection at which time mean bacterial concentration $\left(\log _{10} \mathrm{CFU} / \mathrm{ml}\right)$ ranged from 4.4-4.8 in the blood and from 1.82.3 in the cerebral spinal fluid (CSF). Pre-treatment frequency of bacteremia (100\%) and meningitis (17-23\%) was similar for all experimental groups. Antibiotic concentrations in blood and CSF 2 h after a dose exceeded the $E$. coli minimum inhibitory concentration with the exception of CSF cephalothin, which was undetectable. Moxalactam, ampicillin, and chloramphenicol significantly reduced the incidence of bacteremia and meningitis relative to cephalothin or saline controls $(P<0.02)$. Mortality rates among the former three groups were high (64-82\%) but significantly less than in saline or cephalothin-treated rabbits $(\mathbf{1 0 0 \% )}$. In this neonatal model of fulminant sepsis with early meningitis, moxalactam provided no therapeutic advantage over ampicillin or chloramphenicol.
\end{abstract}

\section{Abbreviations}

BHI, brain heart infusion

CFU, colony forming units

CSF, cerebral spinal fluid

IM, intramuscular

IP, intraperitoneal

MBC, minimum bacteriocidal concentration

MIC, minimum inhibitory concentration

The outcome of human neonatal gram-negative meningitis remains grave. Mortality ranges from $15-30 \%$ and sequelae occur in $30-50 \%$ of survivors $(17,18)$. Poor prognosis has been associated with sub-optimal CSF antibiotic concentrations, persistently positive CSF cultures, and ventriculitis (16-18). Moxalactam, a third generation cephalosporin, has potent activity in vitro against Enterobacteriaceae and penetrates well into CSF (22). Preliminary therapeutic trials in animal and human meningitis have been promising $(22,23)$ and suggest that this antibiotic may be useful in neonatal infection; however, several features of meningitis in newborns suggest there may be a limit to the efficacy of antibiotic therapy alone. Bacteremia is present in approximately two-thirds of neonates with gram-negative meningitis; sepsis, shock and disseminated intravascular coagulation may occur $(8,19)$. Fulminant disease and death during the first $2 \mathrm{~d}$ of therapy is not uncommon $(3,12)$. Immaturity may contribute to the severity of infection due to relative deficiencies in humoral, phagocytic, reticuloendothelial, and com- plement-mediated functions in neonates $(9,11,25)$. Many of these characteristics of neonatal gram-negative meningitis are not addressed in existing animals models $(4,6,10,24)$. We have developed a model of overwhelming $E$. coli $\mathrm{K} 1$ sepsis and early meningitis in infant rabbits and have assessed the clinical and bacteriologic effects of several antibiotics in this infection.

\section{MATERIALS AND METHODS}

Bacteria and animal models. E. coli $\mathrm{K} 1$ (strain Blake), isolated from the CSF of a newborn with meningitis, was stored at $-70^{\circ} \mathrm{C}$ in BHI broth with $20 \%$ glycerol (Difco). For each experiment, an aliquot was thawed, plated on BHI agar, grown overnight at $37^{\circ} \mathrm{C}$ and passed once in mice by IP injection. The mouse heart blood isolate was grown to late logarithmic phase in BHI broth and diluted 1:100 in sterile non-bacteriostatic 0.9\% saline (Scientific Products). A 1-ml inoculum containing approximately $10^{7} \mathrm{E}$. coli $\left(\mathrm{LD}_{50}=2 \times 10^{6} \mathrm{CFU}\right)$ was injected IP into 7 - or 14d-old New Zealand white rabbits (Rich-Glo, El Campo, Texas). The dynamics of bacterial infection were initially determined in nine 7-d-old rabbits. Blood and CSF for quantitative culture were obtained hourly from groups of three rabbits until all animals were dead.

Blood and CSF cultures. Ketamine hydrochloride (Bristol Laboratories, $44 \mathrm{mg} / \mathrm{kg} \mathrm{IM}$ ) was used for sedation of animals during procedures. Cardiac puncture was done to obtain $0.1 \mathrm{ml}$ blood. This was added to $0.9 \mathrm{ml}$ Columbia blood culture broth (Difco) and kept on ice until quantitative cultures could be done (within $2 \mathrm{~h}$ ). Serial 10-fold dilutions of each blood broth mixture were made in sterile saline. Duplicate 50- $\mu$ l samples of each dilution were cultured on EMG-agar (Difco) plates and incubated at $37^{\circ} \mathrm{C}$ overnight. The initial blood culture tubes were also kept at $37^{\circ} \mathrm{C}$ and checked for growth if plate cultures were negative. The minimum number of organisms detectable by this method was $10 \mathrm{CFU} / \mathrm{ml}$, although accurate quantitation was only possible for blood samples with $200 \mathrm{CFU} / \mathrm{ml}$ or more.

CSF was obtained by cisternal puncture using a neonatal scalp vein infusion set with a 25 gauge needle (Argyle, Sherwood Medical, St. Louis, MO). A short length of plastic tubing was connected by luer lock to the infusion set to allow application of gentle mouth suction during the procedure. CSF with visible blood contamination was discarded. Traumatic taps occurred rarely and were usually due to insufficient sedation, or insertion of the needle too deeply. Duplicate 10- $\mu$ l samples of CSF were spread on each of two EMB plates and incubated overnight at $37^{\circ} \mathrm{C}$. Where possible, a single $100-\mu 1$ sample was also plated to increase sensitivity of detection from 100 to $10 \mathrm{CFU} / \mathrm{ml}$. Postmortem cultures were not quantitated unless obtained immediately after death.

Infant rabbits tolerated repeated cardiac and cisternal taps well. In preliminary pharmacokinetic studies in uninfected rabbits, there were no procedure-related deaths even though each animal received up to six cardiac and cisternal punctures over a 12-h period. 
Treatment studies. A total of six antibiotic treatment studies were done with dosage regimens and controls as noted in Table 1. For each study, three to four litters, born within 1-2 d of each other, were used. Littermates were distributed evenly among the groups and matched for weight. Rabbits were infected in groups of five at 10 -min intervals and then returned to their mothers. Four hours later quantitative blood and CSF cultures were obtained from all animals in the same order as that used for IP injection of $E$. coli (pre-therapy culture). Immediately after blood and CSF samples were obtained, each rabbit was given antibiotic or saline $(0.1 \mathrm{ml} / 100 \mathrm{~g})$ subcutaneously. CSF and blood were obtained for antibiotic assay $2 \mathrm{~h}$ after the first dose from five rabbits in each antibiotic group. Animals were kept warm in a $33^{\circ} \mathrm{C}$ incubator until all procedures were finished. All surviving animals had quantitative blood and CSF cultures repeated just before the second dose of antibiotic or saline (post-therapy cultures). Additional cultures were done only if an animal died or if post-therapy cultures were positive. A total of six antibiotic or saline injections were given and animals were observed for 72 $\mathrm{h}$ after completion of therapy.

$M I C / M B C$. Sensitivity of the challenge $E$. coli strain to chloramphenicol (Parke Davis, Morris Plains, NJ), ampicillin (Bristol Laboratories, Syracuse, NY), moxalactam, and cephalothin (Eli Lilly and Co., Indianapolis, IN) was determined by standard tube dilution technique using Mueller-Hinton broth (Difco) and an inoculum of $10^{6} \mathrm{E}$. coli. MIC was defined as the lowest concentration of antibiotic $(\mu \mathrm{g} / \mathrm{ml})$ that inhibited visible growth of $E$. coli after overnight incubation at $37^{\circ} \mathrm{C}$. $\mathrm{MBC}$ was determined by plating $0.01 \mathrm{ml}$ of broth from each tube with no visible bacterial growth on Mueller-Hinton agar and incubating overnight at $37^{\circ} \mathrm{C}$. MBC was defined as the lowest antibiotic concentration $(\mu \mathrm{g} / \mathrm{ml})$ that killed $99.9 \%$ of the original inoculum $(<10$ colonies after overnight incubation).

Antibiotic assays. Bioactive antibiotic concentrations were determined by agar disc diffusion using $150-\mathrm{mm}$ petri dishes and $20-\mu l$ volumes of serum or CSF. Indicator organisms and agar were as follows: $E$. coli (strain Blake) in antibiotic medium \#1 (Difco) for moxalactam; Aeromonas hydrophila (Minnesota \#2 strain, kindly provided by $\mathrm{R}$. Boshard, Clinical Microbiology Laboratories, University of Utah) in Columbia agar (Difco) for chloramphenicol; Bacillus subtilis spores (Difco) in nutrient agar (Difco) for ampicillin and cephalothin (2). Antibiotic standards were included in duplicate on each plate. Serum standards were diluted in pooled normal rabbit serum and CSF standards in phosphate buffered saline, $\mathrm{pH} 7.4$. Sensitivity of the assay ( $\mu \mathrm{g} /$ $\mathrm{ml}$ ) for each antibiotic was the following: moxalactam, 1.0; chloramphenicol, 2.5; ampicillin, 1.0; and cephalothin, 0.5 .

Statistical analysis. Fisher's exact or chi-square tests were used to compare differences in bacteremia, meningitis, and survival between groups. Quantitative blood and CSF bacterial counts were compared using Student's $t$ test.

\section{RESULTS}

Animal model. IP injection of infant rabbits with $10^{7} \mathrm{E}$. coli resulted in a rapidly progressive infection with bacteremia in 9/ 9 animals, meningitis in $7 / 9$ animals and death in all untreated animals. These results were validated in subsequent experiments involving 29 infected rabbits treated with saline. The course of infection was the same for 7- and 14-d-old rabbit pups. The concentration of $E$. coli in the blood remained stable throughout the course of infection whereas there was a gradual increase in $\mathrm{CSF}$ bacterial counts from a mean of $10^{2.6} \mathrm{CFU} / \mathrm{ml}$ at $2 \mathrm{~h}$ to $10^{4.4} \mathrm{CFU} / \mathrm{ml}$ at $8 \mathrm{~h}$ (Fig. 1). The first sign of illness, mucoid green diarrhea, appeared 4-5 h after IP injection. Terminally, pups had progressive hypothermia, lethargy, and hypotonia with slowed respirations. No seizure activity was noted. All deaths in untreated animals occurred between $6-10 \mathrm{~h}$ after IP injection.

Treatment studies. The results of the first treatment experiment are shown in Figure 2. There was a significant reduction in bacteremia and meningitis $(P<0.01)$ and improved survival
Table 1. Antibiotic regimens used to treat $\mathrm{E}$. coli $K 1$ sepsis and meningitis in infant rabbits

\begin{tabular}{rlccc}
\hline Study & \multicolumn{1}{c}{ Antibiotics* } & \# Animals & $\begin{array}{c}\text { Daily } \\
\text { dose } \\
(\mathrm{mg} / \mathrm{kg})\end{array}$ & $\begin{array}{c}\text { Dose } \\
\text { interval } \\
\text { (h) }\end{array}$ \\
\hline 1. & A. Moxlactam & 15 & 30 & 8 \\
& B. Cephalothin & 15 & 75 & 8 \\
2. & A. Moxalactam & 10 & 30 & 8 \\
& B. Ampicillin & 10 & 300 & \\
3. & A. Moxalactam & 14 & 40 & 12 \\
& B. Ampicillin & 14 & 200 & \\
4-6. & A. Moxalactam & 35 & 60 & 12 \\
& B. Chloramphenicol & 51 & 100 & \\
\hline
\end{tabular}

* Each antibiotic given a total of six times. Four to five saline-treated controls included in each experiment.

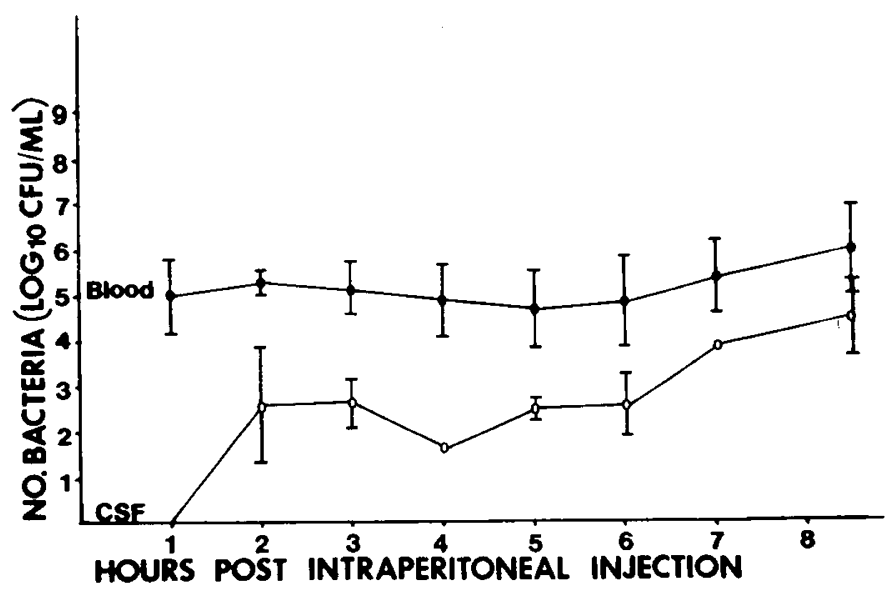

Fig. 1. Hourly quantitative blood and CSF bacterial counts (mean \pm $1 \mathrm{SD}$ ) after IP injection of $10^{7} \mathrm{E}$. coli into 7-d-old rabbits. Three animals were sampled per hour.

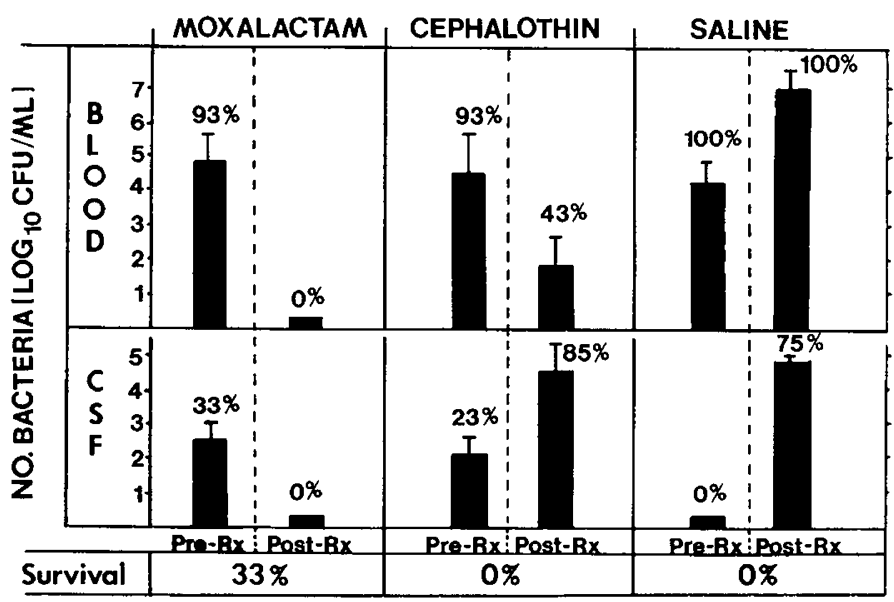

Fig. 2. Incidence $(\%)$ and degree $\left(\log _{10} \mathrm{CFU} / \mathrm{ml}\right)$ of bacteremia and meningitis in infant rabbits with $E$. coli $\mathrm{K} 1$ sepsis and meningitis before and after treatment with moxalactam, cephalothin, or saline. Final outcome $72 \mathrm{~h}$ after discontinuing therapy is indicated as \% survival.

$(P<0.05)$ in animals receiving moxalactam compared with those given cephalothin or saline.

The results of the six treatment studies are shown in Table 2. Before therapy all groups had a similar incidence and degree of bacteremia and meningitis. The mean bacterial concentrations $\left(\log _{10} \mathrm{CFU} / \mathrm{ml}\right)$ ranged from $4.4-4.8$ in the blood and from 1.82.3 in the CSF. The mean $\log _{10} \mathrm{CFU} / \mathrm{ml}( \pm 1 \mathrm{SD})$ of blood was higher in animals with meningitis $(5.2 \pm 0.8)$ than in animals with bacteremia alone $(4.4 \pm 1.0)$, but these differences were not 
statistically significant. A single dose of moxalactam, chloramphenicol, or ampicillin significantly reduced the incidence of bacteremia and meningitis compared with cephalothin or saline $(P<0.02)$. The infrequent positive post-treatment blood and CSF cultures in the moxalactam, chloramphenicol, and ampicillin groups occurred only in rabbits dying after a single antibiotic dose, with one exception. This was in an animal treated with ampicillin who died $3 \mathrm{~d}$ after therapy was discontinued. Postmortem blood and CSF cultures both grew E. coli.

The majority of deaths in all groups occurred within the first $16 \mathrm{~h}$ of infection between the first and second dose of antibiotic or saline. Animals treated with chloramphenicol had a significantly higher early survival rate relative to those receiving moxalactam, cephalothin, or saline $(P<0.02)$. Ampicillin improved early survival relative to the saline group only $(P<0.02)$. There were no differences in terms of overall survival among animals treated with moxalactam, chloramphenicol, or ampicillin. All three groups had a better outcome than saline-treated animals $(P<0.03)$ whereas only the moxalactam and chloramphenicol groups had significantly improved survival compared with the cephalothin group $(P<0.05)$.

The MIC/MBC $(\mu \mathrm{g} / \mathrm{ml})$ for each antibiotic against the challenge $E$. coli strain were the following: moxalactam, $0.06 / 0.06$; ampicillin, 0.5/1.0; cephalothin, $0.5 / 0.5$; and chloramphenicol, 4/128.

Antibiotic concentrations in blood and CSF $2 \mathrm{~h}$ after the first dose are shown in Table 3 . Serum concentrations of all four antibiotics exceeded the $E$. coli MIC by a large margin. Moxalactam, ampicillin, and chloramphenicol achieved CSF concentrations in excess of the MIC but cephalothin was not detectable $(<0.5 \mu \mathrm{g} / \mathrm{ml})$.

\section{DISCUSSION}

Direct intracisternal inoculations of bacteria into adult rabbits is frequently employed to study the penetration of antibiotics into CSF and the associated bacteriologic effects (6). The disadvantages of this model for the study of newborn meningitis include the route of infection, the age of the animals, the low incidence of ventriculitis (7), and the lack of systemic sepsis. Neonatal rat models also have limited value in therapeutic studies because the animals are usually sacrificed in order to sample CSF reliably $(4,10)$. A recently described neonatal guinea pig model (24) proved impractical due to the small litter size (two to four pups per litter) and the long, unpredictable gestation period (59-72 d) of guinea pigs.

We chose to use infant rabbits because their body size allows repeated blood and CSF sampling and similarly aged litters of seven to ten pups are readily available. Other advantages of our model are the young age, the reproducibility of infection and the similarity to severe human disease, in which systemic sepsis with early death is described. A disadvantage in using small animals $(80-150 \mathrm{~g})$ is the limited volume of blood and CSF which can be safely withdrawn over a short period of time. For this reason, we did not attempt to do pharmacokinetics in infected rabbits nor did we follow CSF parameters of inflammation such as cell count, protein, and sugar. The rapid course of infection from initiation to death (usually $<24 \mathrm{~h}$ ) made it unlikely that evidence of meningeal inflammation such as leukocytosis, hypoglycoracchia, or elevated protein would be present (15). To ensure that subcutaneously administered antibiotics were adequately absorbed, a single blood and CSF antibiotic concentration was measured $2 \mathrm{~h}$ after the first dose in five animals from each treatment group. The results shown in Table 3 indicate that therapeutic serum and CSF antibiotic concentrations were achieved with all drugs except cephalothin in CSF. The low CSF/ serum antibiotic concentration ratios observed for moxalactam and ampicillin probably reflect a lack of CNS inflammation $6 \mathrm{~h}$ after initiation of infection. The overwhelming nature of the untreated infection in our model made it essential to assess whether the presence of meningitis was a factor in mortality; hence, we first compared moxalactam with cephalothin. Because

Table 2. Antibiotic treatment of $\mathrm{E}$. coli $K 1$ sepsis and meningitis in infant rabbits*

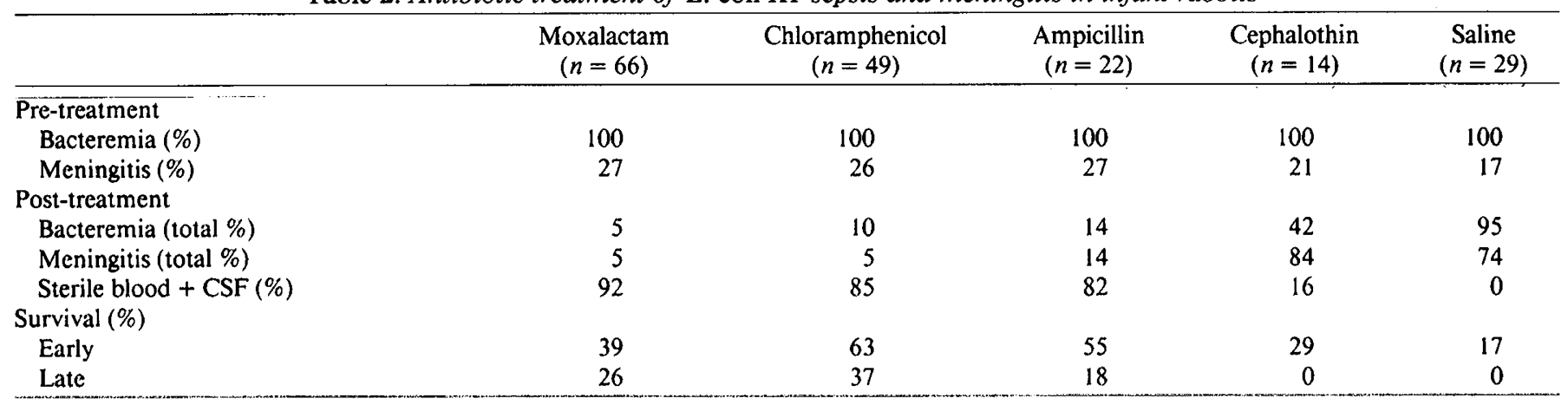

* The timing of pre- and post-treatment cultures is indicated in the text. Early survival represents the animals alive at the time of the second dose of antibiotic or saline. Late survival represents the animals alive $72 \mathrm{~h}$ after completing therapy.

Table 3. Antibiotic concentrations in serum and cerebral spinal fluid (CSF) $2 \mathrm{~h}$ after the first dose in infant rabbits with $\mathrm{E}$. coli $K 1$ sepsis and meningitis

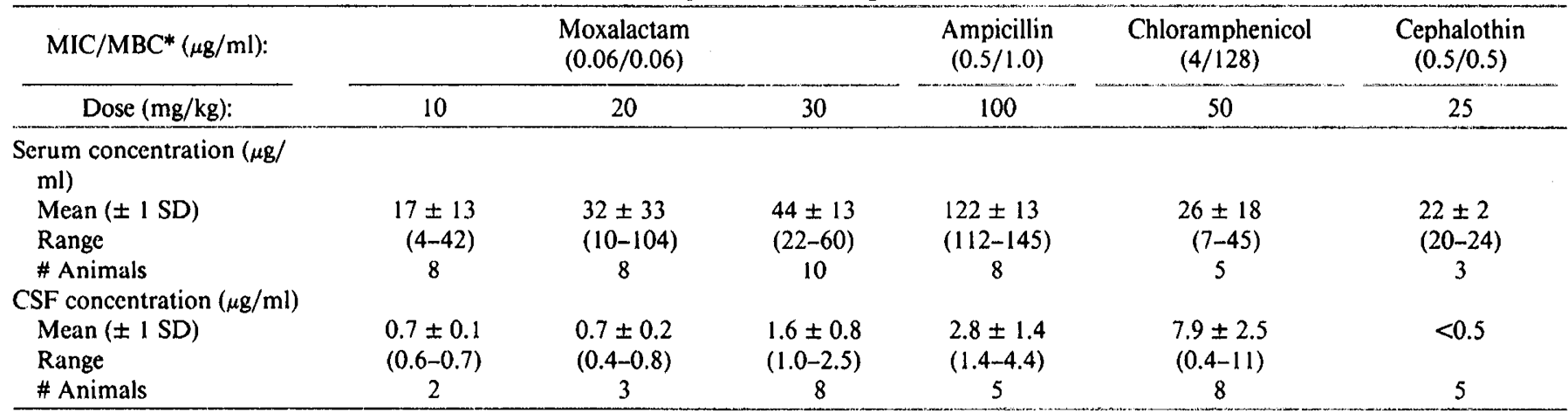

* MIC/MBC, minimum inhibitory concentration/minimum bacteriocidal concentration. 
cephalothin is usually not detectable in CSF, we expected that differences in outcome between the two treatment groups would relate to antibiotic efficacy in sterilizing CSF. Indeed, despite excellent serum cephalothin concentrations, none was detectable in CSF. At the time cephalothin therapy was initiated ( $4 \mathrm{~h}$ after IP infection), only $21 \%$ of the rabbits had positive CSF cultures. The fact that $85 \%$ of the group developed and subsequently died with meningitis suggests that seeding of the CNS with bacteria occurred in the majority of rabbits before the first antibiotic dose. The low yield of positive CSF cultures early in the course of infection may have been due to colony counts less than $10^{2}$ $\mathrm{CFU} / \mathrm{ml}$ in cisternal CSF. The outcome in animals treated with cephalothin has a human clinical parallel in the occurrence of "partly treated" meningitis when drugs with limited ability to penetrate CSF are used to treat bacteremic infections (14).

Moxalactam, chloramphenicol, and ampicillin were equally effective in sterilizing the blood and CSF. The therapeutic response to chloramphenicol was surprising in view of its bacteriostatic activity against Enterobacteriaceae (20). Results have been equivocal $(1,21,26)$ using chloramphenicol in both clinical and animal studies of gram-negative meningitis. In our study, none of the surviving rabbits treated with chloramphenicol (100 $\mathrm{mg} / \mathrm{kg} /$ day) relapsed, although the observation period of $72 \mathrm{~h}$ may not have been long enough. The low CSF bacterial concentrations at the beginning of therapy may also have been a factor in the demonstrated efficacy of chloramphenicol; however, we have found that chloramphenicol is bacteriostatic against this strain of $E$. coli even when tested against an inoculum of $10^{2}$ $\mathrm{CFU}(\mathrm{MIC} / \mathrm{MBC}=2 / 64 \mu \mathrm{g} / \mathrm{ml})$. Survival in the first $16 \mathrm{~h}$ of infection was significantly better with chloramphenicol than with moxalactam. One might speculate that the bactericidal activity of moxalactam may have produced rapid in vivo bacteriolysis with release of toxic bacterial products such as endotoxin. This would not be expected with a bacteriostatic drug such as chloramphenicol.

Despite rapid sterilization of blood and CSF by moxalactam, chloramphenicol, or ampicillin, the mortality rates in our model ranged from $64-82 \%$. These results suggest that antibiotic therapy alone may have limited efficacy in preventing many of the early deaths seen in fulminant neonatal gram-negative meningitis. Recent studies have demonstrated that white cell transfusions and fresh frozen plasma may improve survival in neonatal sepsis and meningitis $(5,13)$. Specific antibody to surface antigens of gram-negative bacteria and drugs with anti-endotoxin or antiinflammatory activity should also be evaluated. Our model provides an appropriate system for examining the efficacy of such adjunctive therapies.

\section{REFERENCES AND NOTES}

1. Beam, T. R., Jr. and Allen, J. C.: Comparison of cefamandole, cephalothin, ampicillin, and chloramphenicol in experimental Escherichia coli meningitis. Antimicrob. Ag. Chemother., 17: 37 (1980)

2. Bennett, J. V., Brodie, J. L., Benner, E. J., and Kirby, W. M. M.: Simplified accurate method for antibiotic assay of clinical specimens. Appl. Microbiol., 14: $170(1966)$

3. Berman, P. H. and Banker, B. Q.: Neonatal meningitis. A clinical and pathological study of 29 cases. Pediatrics, 38: 6 (1966).
4. Bortolussi, R., Ferrieri, P., and Wannamaker, L. W.: Dynamics of Escherichia coli infection and meningitis in infant rats. Immun., 22: 480 (1978).

5. Bortolussi, R., Issekutz, A. C., and Barnard, D. R.: The use of plasma infusion for neonatal sepsis. Pediatr Res., 15: 607 (1981).

6. Dacey, R. G. and Sande, M. A.: Effect of probenecid on cerebrospinal fluid concentrations of penicillin and cephalosporin derivatives. Antimicrob. Ag. Chemother., 6: 437 (1974).

7. Dacey, R. G., Welsh, J. E., Scheld, W. M., Winn, H. R., Jane, J. A., and Sande M. A.: Alterations in cerebrospinal fluid outflow resistance in experimental bacterial meningitis. Trans. Am. Neurol. Assoc., 103: 142 (1978).

8. Fosson, A. R. and Fine, R. N.: Neonatal meningitis: presentation and discussion of 21 cases. Clin. Pediatr., 7: 404 (1968).

9. Freedman, R. M., Johnston, D., Mahoney, M. J., and Pearson, H. A.: Development of splenic reticuloendothelial function in neonates. J. Pediatr., 96: 466 (1980).

10. Glode, M. P., Sutton, A., Moxon, E. R., and Robbins, J. B.: Pathogenesis of neonatal Escherichia coli meningitis: induction of bacteremia and meningitis in infant rats fed $E$. coli $\mathrm{K} 1$. Infect. Immun., 16: 75 (1977).

11. Gotoff, S. P.: Neonatal immunity. J. Pediatr., 85: 149 (1974).

12. Heckmatt, J. Z.: Coliform meningitis in the newborn. Arch. Dis. Child., 51: 569 (1976)

13. Laurenti, F., Ferro, R., Isacchi, G., Panero, A., Savignoni, P. G., Malignino, F., Palermo, D., Mandelli, F., and Bucci, G.: Polymorphonuclear leukocyte transfusion for the treatment of sepsis in the newborn infant. J. Pediatr., 98: 118 (1981).

14. Mangi, R. J., Kundargi, R. S., Quintiliani, R., and Andriole, V. T.: Develop ment of meningitis during cephalothin therapy. Ann. Intern. Med., 78: 347 (1973).

15. McAllister, C. K., O'Donoghue, J. M., and Beaty, H. N.: Experimental pneumococcal meningitis. II. Characterization and quantitation of the inflammatory process. J. Infect. Dis., 132: 355 (1975).

16. McCracken, G. H., Jr.: The rate of bacteriologic response to antimicrobial therapy in neonatal meningitis. Amer. J. Dis. Child., I23: 547 (1972).

17. McCracken, G. H., Jr. and Mize, S. G.: A controlled study of intrathecal antibiotic therapy in gram-negative enteric meningitis of infancy. J. Pediatr. 89: 66 (1976).

18. McCracken, G. H., Jr., Mize, S. G., and Threlkeld, N.: Intraventricular gentamicin therapy in gram-negative bacillary meningitis of infancy. Lancet, l: 787 (1980).

19. Overall, J. C., Jr.: Neonatal bacterial meningitis. Analysis of predisposing factors and outcome compared with matched control subjects. J. Pediatr., 76: 499 (1970)

20. Rahal, J. J., Jr. and Simberkoff, M. S.: Bactericidal and bacteriostatic action of chloramphenicol against meningeal pathogens. Antimicrob. Ag. Chemother., 15: 13 (1979).

21. Rahal, J. J. and Simberkoff, M. S.: Host defense and antimicrobial therapy in adult gram-negative bacillary meningitis. Ann. Intern. Med., 96: 468 (1982).

22. Schaad U. B McCracken, G. H Jr Loock, C.A and Thomas, M I Pharmacokinetics and bacteriologic efficacy of moxalactam, cefotaxime, cefoperazone, and rocephin in experimental bacterial meningitis. J. Infect. Dis., 143: 156 (1981).

23. Schaad, U. B., McCracken, G. H., Jr., Threlkeld, N., and Thomas, M. L.: Clinical evaluation of a new broad-spectrum oxa-beta-lactam antibiotic, moxalactam, in neonates and infants. J. Pediatr., 98: 129 (1981).

24. Sinai, R. E., Marks, M. I., Powell, K. R., and Pai, C. H.: Model of neonatal meningitis caused by Escherichia coli $\mathrm{K} 1$ in guinea pigs. J. Infect. Dis., 141: 193 (1980).

25. Stiehm, E. R., Winter, H. S., and Bryson, Y. J.: Cellular (T cell) immunity in the human newborn. Pediatrics, 64: 814 (1979).

26. Strausbaugh, L. J. and Sande, M. A.: Factors influencing the therapy of experimental Proteus mirabilis meningitis in rabbits. J. Infect. Dis., 137: 251 (1978).

27. Dr. Law is a Fellow of the Medical Research Council of Canada. Her present address: Infectious Disease Research Laboratory, Izaak Walton Killam Hospital for Children, 5850 University Avenue, Halifax, Nova Scotia, B3J 3G9, Canada.

28. Requests for reprints should be addressed to: Dr. Melvin I. Marks, Pediatric Infectious Diseases, University of Oklahoma, Health Sciences Center, P.O. Box 26901, Oklahoma City, Oklahoma 73190.

29. Received for publication November $16,1982$.

30. Accepted for publication June 2, 1983. 